Pacific Journal of Mathematics

A QUASI-ADDITIVITY TYPE CONDITION AND THE
INTEGRAL OVER A BY VARIETY 


\title{
A QUASI-ADDITIVITY TYPE CONDITION AND THE INTEGRAL OVER A BV VARIETY
}

\author{
Primo Brandi and AnNa Salvadori
}

\begin{abstract}
The aim of the present paper is that of developing a theory for the Weierstrass-Cesari integral of the calculus of variations $\mathscr{J}=$ $\int_{T} F(p, q)$ over a variety $T$ (not necessarily continuous nor Sobolev's), with respect to a nonlinear parametric integrand $F(p, q)$. As is well known, in the case of continuous BV varieties, Cesari framed the integral $\mathscr{J}$ in an abstract, general setting by means of the Burkill-Cesari integration process on a "quasi-additive" set function. We introduce here a suitable condition of quasi-additivity type for a couple of set functions (the $\Gamma$-quasi additivity) which allows to adopt Cesari formulation, even in this more general situation and represents the key-idea for the outcome of our research.
\end{abstract}

1. Introduction. In [16] Cesari established a very general axiomatization of the non-linear Weierstrass-type integral $\mathscr{I}=\int_{T} F(p, q)$ over a variety $T$. Indeed, first Cesari introduced the concepts of quasi-additivity for set functions $\phi(I)$ and of an integral of such set functions, now usually called the Burkill-Cesari integral and denoted $\mathrm{BC} \int \phi$, an extension of the classical Burkill process. Then, for any given set function $\phi(I)$ and continuous map $T$, Cesari considered the set function $\Phi(I)=F(T(\omega(I)), \phi(I))$, where $\omega$ is a choice function, i.e. $\omega(I) \in I$, and proved that, if $T$ is any continuous map, $F$ is any parametric integrand, and $\phi$ is quasi-additive and BV, then also $\Phi$ is quasi-additive and BV. In other words, the non-linear transformation $F$ preserves quasi-additivity and bounded variation, and then the Weierstrass-type integral $\mathscr{I}=\int_{T} F(p, q)$ can be defined as the Burkill-Cesari integral

$$
\mathscr{I}=\mathrm{BC} \int \Phi(I)
$$

and can be computed by a process of limit which is an extension of the classic Burkill integral. Cesari proved a number of properties of the Weierstrass-type integral $\mathscr{I}=\mathrm{BC} \int \Phi(I)$ [16]. Moreover, for functions $\phi$ with values in $R^{n}$ and under mild assumptions, Cesari proved 
further that the integral $\mathrm{BC} \int \phi(I)$ defines a non-negative measure $\mu$ and a vector measure $\nu$ such that the Radon-Nikodym derivative $\theta(s)=d \nu / d \mu$ behaves as a generalized Jacobian vector, and $\mathscr{I}$ has a representation as a Lebesgue-Stieltjes integral [17]

$$
\mathscr{I}=\mathrm{BC} \int \Phi(I)=\int_{T} F(p, q)=\int F(T(s), \theta(s)) d \mu
$$

We extended this representation statement to the case of $\phi$ with values in any reflexive Banach space, and to do this we used the concept of martingales from random analysis [6, 7].

Successively many authors studied the integral $\mathrm{BC} \int \Phi(I)$, both in the parametric and in the non-parametric cases, established further properties of the integral and showed that a number of recent processes of integration can be framed in terms of quasi-additive set functions and related Burkill-Cesari integral (see [21] for a survey).

Note that, if the integrand does not depend explicitly on the variety, i.e. is of the type $F(q)$, then it is possible to extend the definition of Weierstrass integrals over BV curves and surfaces $T$-not necessarily continuous-in terms of quasi-additive set functions $\Phi$ (cf. $[1,20$, $8,10,11,12])$. The continuity assumption on the variety $T$ has not been removed yet in the general case $F(p, q)$.

The aim of the present paper is to extend the definition of $\mathscr{I}$ to the case of $\mathrm{BV}$ varieties $T$, possibly discontinuous, and complete integrand functions $F(p, q)$. To do this we deepen the study we started in [11]. Namely, first we avoid the use of a choice function $\omega$, and replace the term $T(\omega(I))$ by an arbitrary set function $p(I)$. Then we introduce a new condition of quasi-additivity type, on the couple $(p, \phi)$ that we call $\Gamma$-quasi-additivity. We are able to prove that, if $(p, \phi)$ is $\Gamma$-quasi-additive and $\phi$ is $\mathrm{BV}$, then the set function $\Phi(I)=F(p(I), \phi(I))$ is quasi-additive and BV, and thus a Weierstrass type integral $\mathscr{I}=\int_{T} F(p, q)$ can still be defined as a Burkill-Cesari integral BC $\int \Phi(I)$, even for BV curves and surfaces, possibly discontinuous, not Sobolev's, and complete integrands $F(p, q)$. Note that the new global condition is weaker than the couple of assumptions: continuity on $T$ and quasi-additivity on $\phi$.

After the preliminaries $(\S 2)$, we prove the statement above which guarantees the existence of the integral $\mathrm{BC} \int \Phi$, and in $\S 3$ we compare the new condition of quasi-additivity with previous conditions. 
In $\S 4$ we prove that, even in the new general situation, the integral BC $\int \Phi$ has a Lebesgue-Stieltjes integral representation in terms of a finite measure $\mu$, a vector measure $\nu$ and Radon-Nikodym derivative $d \nu / d \mu$, extending therefore Cesari's results in [17], as well our previous results in $[6,7]$. Finally, in $\S 5$ we present some applications of the previous discussion on the integrals of the calculus of variations over a BV curve, also in the presence of a BV weight (not necessarily continuous nor positive).

We wish to mention that in [13] we carried over analogous results for the nonparametric Weierstrass-type integral. Moreover in [14] we established the lower semicontinuity, both for the parametric and the non-parametric Weierstrass-integral in terms of a global convergence, for couples of sets functions $\left(p_{n}, \phi_{n}\right)$ which is inspired to the present $\Gamma$-quasi-additivity. This result found application to $L_{1}$-convergence of equi BV varieties.

2. Preliminaries. Let $(A, \mathscr{G})$ be a topological space, we denote by $\mathscr{M}$ the family of all the subsets of $A$ and by $\mathscr{B}$ the $\sigma$-algebra generated by $\mathscr{G}$. We consider a subfamily $\{I\} \subset \mathscr{M}$ and call intervals the sets $I$. A finite system $D=\left[I_{1}, \ldots, I_{N}\right]$ is a finite collection of non-overlapping intervals, i.e.

$$
I_{i}^{0} \neq \varnothing \quad \text { and } \quad I_{i}^{0} \cap \bar{I}_{j}=\varnothing, \quad i \neq j, i, j=1, \ldots, N .^{1}
$$

Let $(T, \gg)$ be a directed set and let $\left(D_{t}\right)_{t \in T}$ be a given net of finite systems. Let $s: \mathscr{M} \times \mathscr{M} \rightarrow\{0,1\}$ be the function defined by : $s(H, K)=1$ when $H \subset K, s(H, K)=0$ otherwise.

Let $\phi:\{I\} \rightarrow E$ be a given interval function, where $E$ is a real Banach space.

The function $\phi$ is said to be Burkill-Cesari integrable (BC-integrable) ([16]) over $M \in \mathscr{M}$ if the limit below exists

$$
\lim _{T} \sum_{I \in D_{t}} s(I, M) \phi(I)
$$

in this case, we shall denote by $\mathrm{BC} \int_{M} \phi$ its value.

\footnotetext{
${ }^{1}$ Where $I^{0}$ and $\bar{I}$ denote the $G$-interior and the $G$-closure of $I$, respectively.
} 
The function $\phi$ is said to be of bounded variation (BV) over $M \in \mathscr{M}$ if

$$
V(\phi, M)=\varlimsup_{T} \sum_{I \in D_{t}} s(I, M)\|\phi(I)\|<+\infty
$$

The function $\phi$ is said to be quasi-additive (q.a.) ([16]) over $M \in \mathscr{M}$ if

(q.a.) given $\varepsilon>0$ there exists $t_{1}=t_{1}(M, \varepsilon)$ such that, for every $t_{0} \gg t_{1}$ there exists $t_{2}=t_{2}\left(M, \varepsilon, t_{0}\right)$ such that, if $t \gg t_{2}$ then

(i) $\sum_{I} s(I, M)\left\|\sum_{J} s(J, I) \phi(J)-\phi(I)\right\|<\varepsilon$,

(ii) $\sum_{J} s(J, M)\left[1-\sum_{I} s(J, I) s(I, M)\right]\|\phi(J)\|<\varepsilon$

where $D_{t_{0}}=[I], D_{t}=[J]$.

The following results are well known $([16,15,22,4])$.

$\mathrm{P}_{1}$. If $\phi$ is q.a. on $M$, then it is BC-integrable over $M$.

$\mathrm{P}_{2}$. If $\phi$ is q.a. and $B V$ on $M$, then $\|\phi\|$ is q.a. on $M$.

$\mathrm{P}_{3}$. If $\phi$ is q.a. and $B V$ on $M$, then $\phi$ is q.a. on $M$ for every $M \in \mathscr{M}$.

3. Existence of the integral $\mathrm{BC} \int_{A} F(p, \phi)$. Let $(K, d)$ be a metric space, $E$ be a uniformly convex Banach space and $B$ be a real Banach space.

We consider the following functions:

$$
F: K \times E \rightarrow B, \quad p:\{I\} \rightarrow K \quad \text { and } \quad \phi:\{I\} \rightarrow E
$$

and denote by $\Phi:\{I\} \rightarrow B$ the set function defined by

$$
\Phi(I)=F(p(I), \phi(I))
$$

Following Cesari ([16]), the BC-integral of the function $\Phi$, when it exists, will be called the parametric Weierstrass integral of the Calculus of Variations (W-integral) and denoted by $\mathrm{BC} \int_{A} F(p, \phi)$.

Thus, any sets of conditions guaranteeing that $\Phi$ is q.a. and BV is an existence theorem for the integral $\mathrm{BC} \int_{A} F(p, \phi)$.

All throughout the paper, we will suppose that the integrand $F$ satisfies the following conditions:

$\left(\mathrm{F}_{1}\right) \quad F$ is bounded on $K \times S_{1}$, where $S_{1}=\{x \in E:\|x\|=1\}$;

$\left(\mathrm{F}_{2}\right) \quad F$ is uniformly continuous on $K \times S_{1}$;

$\left(\mathrm{F}_{3}\right) \quad F(k, t x)=t F(k, x)$, for every $t \geq 0,(k, x) \in K \times E$. 
We recall here that the function $p$ is said to satisfy the condition $(\gamma)([2,9])$ if

$(\gamma)$ given $\varepsilon>0$ there exists $t_{1}=t_{1}(\varepsilon)$ such that for every $t_{0} \gg t_{1}$ there exists $t_{2}=t_{2}\left(\varepsilon, t_{0}\right)$ such that, if $t \gg t_{2}$ then

$$
\max _{I} \max _{J \subset I} d(p(J), p(I))<\varepsilon
$$

where $D_{t_{0}}=[I], D_{t}=[J]$.

The following existence theorem for the parametric $\mathrm{W}$-integral is well known $([16,23,9])$.

\section{THEOREM 3.1. Suppose that}

$F$ satisfies conditions $\left(F_{1}\right),\left(F_{2}\right)$ and $\left(F_{3}\right)$;

$p$ satisfies condition $(\gamma)$;

$\phi$ is q.a. and $B V$ on $A$;

then the function $\Phi$ is q.a. and $B V$ on $A$.

We propose now a joint condition on the couple of set functions $(p, \phi)$ that will lead to an improvement of this result.

Definition 3.2. We say that the couple $(p, \phi)$ is $\Gamma$-quasi-additive (Г-q.a.) if

( $\Gamma$-q.a.) given $\varepsilon>0$, there exist $0<\sigma=\sigma(\varepsilon) \leq \varepsilon$ and $t_{1}=t_{1}(\varepsilon)$ such that for every $t_{0} \gg t_{1}$ there exists $t_{2}=t_{2}(\varepsilon)$ such that if $t \gg t_{2}$ then

(i) $\sum_{I}\left\|\sum_{J \in \Gamma_{I}} \phi(J)-\phi(I)\right\|<\varepsilon$,

(ii) $\sum_{I}\left\|\sum_{J \notin \Gamma_{I}} s(J, I) \phi(J)\right\|<\varepsilon$,

(iii) $\sum_{J}\left[1-\sum_{I} s(J, I)\right]\|\phi(J)\|<\varepsilon$

where $D_{t_{0}}=[I], D_{t}=[J]$ and $\Gamma_{I}$ is a subfamily (even empty) of the set $\{J \subset I: d(p(I), p(J))<\sigma\}$.

The following propositions, which can be easily proved, point out the connections between the new condition ( $\Gamma$-q.a.) and the previous ones, i.e. $(\gamma)$ and (q.a.).

$\mathbf{P}_{4}$. If $\phi$ is q.a. on $A$ and $p$ satisfies condition $(\gamma)$, then the couple $(p, \phi)$ is $\Gamma$-q.a.

$\mathrm{P}_{5}$. If the couple $(p, \phi)$ is $\Gamma$-q.a., then $\phi$ is q.a. on $A$. 
However note that condition ( $\Gamma$-q.a.) does not necessarily imply that $p$ satisfies condition $(\gamma)$, as the applications will show (see $\S 5$ ).

In the following we shall need the result below.

Proposition 3.3. If $(p, \phi)$ is $\Gamma$-q.a. and $\phi$ is $B V$, then the couple $(p,\|\phi\|)$ is $\Gamma$-q.a., with the same $\sigma=\sigma(\varepsilon)$ and $\Gamma_{I}, I \in\{I\}$.

Proof. Let $\varepsilon>0$ be fixed and let $t_{0}, t \in T$ be given, corresponding to $\frac{\varepsilon}{4}$, in force of condition ( $\Gamma$-q.a.).

By virtue of $P_{5}$ and $P_{2}$, we can suppose without loss of generality that

$$
\left|\sum_{I}\|\phi(I)\|-V(\phi, A)\right|<\frac{\varepsilon}{4} \quad \text { and } \quad\left|\sum_{J}\|\phi(J)\|-V(\phi, A)\right|<\frac{\varepsilon}{4},
$$

where $D_{t_{0}}=[I]$ and $D_{t}=[J]$. Then we have ${ }^{2}$

$$
\begin{aligned}
& \sum_{I} \mid \sum_{J \in \Gamma_{I}}\|\phi(J)\|-\|\phi(I)\|+\sum_{I}\left[\sum_{J \notin \Gamma_{I}} s(J, I)\|\phi(J)\|\right] \\
& \quad+\sum_{J}\left[1-\sum_{I} s(J, I)\right]\|\phi(J)\| \\
& =\sum_{I}\left[\sum_{J \in \Gamma_{I}}\|\phi(J)\|-\|\phi(I)\|\right]+2 \sum_{I}\left[\sum_{J \in \Gamma_{I}}\|\phi(J)\|-\|\phi(I)\|\right]^{-} \\
& \quad+\sum_{I}\left[\sum_{J \notin \Gamma_{I}} s(J, I)\|\phi(J)\|\right]+\sum_{J}\left[1-\sum_{I} s(J, I)\right]\|\phi(J)\| \\
& \leq \sum_{J}\|\phi(J)\|-\sum_{I}\|\phi(I)\|+2 \sum_{I}\left\|\sum_{J \in \Gamma_{I}} \phi(J)-\phi(I)\right\| \\
& \quad<\left|\sum_{J}\|\phi(J)\|-V(\phi, A)\right|+\left|\sum_{I}\|\phi(I)\|-V(\phi, A)\right|+2 \frac{\varepsilon}{4}<4 \frac{\varepsilon}{4}=\varepsilon,
\end{aligned}
$$

which proves the proposition.

\footnotetext{
${ }^{2}$ Where $m^{+}=\max (m, 0)$ and $m^{-}=\max (-m, 0), m \in \mathbb{R}$.
} 
The main result of this section is the following existence theorem which improves (3.1). For the convenience of the reader we recall the result below, due to Warner ([23]) which will be used in the proof.

LEMMA 1.1 OF [23]. Let $\varepsilon>0$ be fixed. Then a positive number $H=H(\varepsilon)$ exists such that, given two finite subsets of $E\left\{e_{i}, i=\right.$ $1, \ldots, n$ and $\left.e_{j}^{\prime}, j=1, \ldots, m\right\}$ and a map $\mathscr{T}$ from $\{1, \ldots, n\}$ into the subsets of $\{1, \ldots, m\}$, we have

$$
\begin{aligned}
\frac{\varepsilon^{2}}{2 H^{2}} \sum_{i=1}^{n} \sum_{+}^{i}\left\|e_{j}^{\prime}\right\| \leq & \sum_{i=1}^{n}\left\|e_{i}-\sum_{j \in \mathcal{F}(i)} e_{j}^{\prime}\right\| \\
& +\sum_{i=1}^{n}\left|\left\|e_{i}\right\|-\sum_{j \in \mathcal{F}(i)}\left\|e_{j}^{\prime}\right\|\right|,
\end{aligned}
$$

where $\sum_{+}^{i}$ denotes the summation relative to the indices $j \in \mathscr{J}(i)$ such that

$$
\left\|\frac{e_{i}}{\left\|e_{i}\right\|}-\frac{e_{j}^{\prime}}{\left\|e_{j}^{\prime}\right\|}\right\| \geq \varepsilon .
$$

THEOREM 3.4. Suppose that $F$ satisfies conditions $\left(\mathrm{F}_{1}\right),\left(\mathrm{F}_{2}\right)$ and $\left(\mathrm{F}_{3}\right)$; the couple $(p, \phi)$ is $\Gamma$-q.a. and $\phi$ is $B V$; then the function $\Phi$ is q.a. and $B V$ on $A$.

Proof. Let $\varepsilon>0$ be fixed. Then, by force of $\left(\mathrm{F}_{2}\right)$, there is $\tau=$ $\tau(\varepsilon / 6 V)>0$ such that $\left|F(p, x)-F\left(p^{\prime}, x^{\prime}\right)\right| \leq \varepsilon / 6 V$ whenever $p, p^{\prime} \in$ $K, x, x^{\prime} \in S_{1}$ with $d\left(p, p^{\prime}\right)+\left\|x-x^{\prime}\right\| \leq r$, where $V=V(\phi, A)$. Let $H=H(\tau)>0$ be the number determined in force of the Lemma 1.1 of [23]. If $M>0$ is such that $|F(k, x)|<M,(k, x) \in K \times S_{1}$ (see $\left.\left(\mathrm{F}_{1}\right)\right)$, we put

$$
\bar{\varepsilon}=\min \left(\tau, \varepsilon / 6 M, \varepsilon \tau^{2} / 48 H^{2} M\right) .
$$

Now let $\sigma=\sigma(\bar{\varepsilon}) \leq \bar{\varepsilon}$ and $t_{1}=t_{1}(\bar{\varepsilon})$ be determined by ( $\Gamma$-q.a.) and for every $t_{0} \gg t_{1}$ let $t_{2}=t_{2}\left(t_{0}, \bar{\varepsilon}\right)$ be also given by ( $\Gamma$-q.a.). Fix $t \gg t_{2}$ and let $D_{t_{0}}=[I], D_{t}=[J]$. If we denote by $\alpha(I)=$ $\phi(I) /\|\phi(I)\|$ and $\beta(J)=\phi(J) /\|\phi(J)\|$ (where $\alpha(I)=0$ if $\phi(I)=0$ and analogously for $\beta$ ), then from Proposition 3.3 above and the 
Lemma 1.1 of [23] we have

$$
\begin{aligned}
& \text { (1) } \sum_{I}\left|\sum_{J \in \Gamma_{I}} \Phi(J)-\Phi(I)\right| \\
& =\sum_{I}\left|\sum_{J \in \Gamma_{I}} F(p(J), \alpha(J))\|\phi(J)\|-F(p(I), \alpha(I))\|\phi(I)\|\right| \\
& \leq \sum_{I}|F(p(I), \alpha(I))|\left|\sum_{J \in \Gamma_{I}}\|\phi(J)\|-\|\phi(I)\|\right| \\
& +\sum_{I} \sum_{J \in \Gamma_{I}}|F(p(J), \beta(J))-F(p(I), \alpha(I))|\|\phi(J)\| \\
& \leq M \bar{\varepsilon}+\frac{\varepsilon}{6 V} \sum_{I}\left[\sum_{J \in \Gamma_{I},\|\beta(J)-\alpha(I)\|<\tau}\|\phi(J)\|\right] \\
& +2 M \sum_{I}\left[\sum_{J \in \Gamma_{I},\|\beta(J)-\alpha(I)\| \geq \tau}\|\phi(J)\|\right] \\
& \leq M \frac{\varepsilon}{6 M}+\frac{\varepsilon}{6 V} V \\
& +2 M \frac{2 H^{2}}{\tau^{2}}\left[\sum_{I}\left\|\sum_{J \in \Gamma_{I}} \phi(J)-\phi(I)\right\|\right. \\
& \left.+\sum_{I}\left|\sum_{J \in \Gamma_{I}}\|\phi(J)\|-\|\phi(I)\|\right|\right] \\
& \leq \frac{\varepsilon}{6}+\frac{\varepsilon}{6}+2 M \frac{2 H^{2}}{\tau^{2}} 2 \frac{\varepsilon \tau^{2}}{48 H^{2} M}=\frac{\varepsilon}{6}+\frac{\varepsilon}{6}+\frac{\varepsilon}{6}=\frac{\varepsilon}{2} \text {. }
\end{aligned}
$$

Moreover we have

$$
\begin{aligned}
& \sum_{I}\left|\sum_{J \notin \Gamma_{I}} s(J, I) \Phi(J)\right| \\
& \quad \leq \sum_{I} \sum_{J \notin \Gamma_{I}} s(J, I)|F(p(J), \beta(J))|\|\phi(J)\| \leq M \bar{\varepsilon}<\frac{\varepsilon}{2}
\end{aligned}
$$

Thus from (1) and (2) the first condition (i) of the q.a. of $\Phi$ is verified. 
Finally, from

$$
\begin{aligned}
\sum_{J}\left[1-\sum_{I} s(J, I)\right]\|\Phi(J)\| \\
\quad \leq M \sum_{J}\left[1-\sum_{I} s(J, I)\right]\|\phi(J)\|<M \bar{\varepsilon}<\varepsilon,
\end{aligned}
$$

the q.a. of $\Phi$ is completely proved.

Furthermore, since $\|\Phi(I)\| \leq M\|\phi(I)\|$ (by $\left(\mathrm{F}_{3}\right)$ ), the function $\Phi$ is trivially $\mathrm{BV}$.

Note that, by force of $\mathrm{P}_{4}$, Theorem 3.4 improves the previous existence result (Theorem 3.1). The applications given in $\S 5$ will emphasize the value of this result.

REMARK 3.5. Let $p^{\prime}:\{I\} \rightarrow K$ be another set function and suppose that $p$ and $p^{\prime}$ satisfy the condition

(i) given $\varepsilon>0$ there exists $t_{1}=t_{1}(\varepsilon)$ such that for every $t \gg t_{1}$ we have

$$
\max _{I \in D_{t}} d\left(p(I), p^{\prime}(I)\right)<\varepsilon .
$$

It is easy to see that, if $(p, \phi)$ is $\Gamma$-q.a., then also $\left(p^{\prime}, \phi\right)$ is $\Gamma$-q.a., and moreover we have that $\mathrm{BC} \int_{A} F(p, \phi) \equiv \mathrm{BC} \int_{A} F\left(p^{\prime}, \phi\right)$.

Thus in the particular case (see [9]) where $p(I)=P(\omega(I))$ and $p^{\prime}(I)=P\left(\omega^{\prime}(I)\right)$, i.e. the set function $p$ comes from the composition of a point function $P: A \rightarrow K$ and a choice function $\omega:\{I\} \rightarrow A$ (with $\omega(I) \in I)$, then the $W$-integral is independent from the choice function $\omega$, within a class of choices which satisfy invariance condition (i).

Moreover in [23] Warner proposes an invariance condition for Wintegrals which contains, as a particular case, the Fréchet equivalence condition for parametric curves and surfaces. Note that Warner's condition can be easily carried over the present setting.

REMARK 3.6. In [9, 10, 11] following an idea developed by Kaiser ([18]) for the variation, we introduced the weighted $\mathrm{W}$-integral. We recall here its definition.

Let $e_{1} \cdot e_{2}: \mathbb{R}^{m} \times \mathbb{R}^{n} \rightarrow E$ be a bilinear form such that $\left\|e_{1} \cdot e_{2}\right\| \leq$ $L\left\|e_{1}\right\|\left\|e_{2}\right\|$, with $L>0, e_{1} \in \mathbb{R}^{m}, e_{2} \in \mathbb{R}^{n}$. We consider the functions:

$$
F: K \times E \rightarrow B, \quad p:\{I\} \rightarrow K, \quad \phi:\{I\} \rightarrow \mathbb{R}^{n}, \quad q:\{I\} \rightarrow \mathbb{R}^{m}
$$


and define the interval function $\Phi_{q}:\{I\} \rightarrow F$ by

$$
\Phi_{q}(I)=F(p(I), \phi(I) \cdot q(I)) .
$$

The BC-integral of $\Phi_{q}$, when it exists, will be called the weighted parametric Weierstrass integral of the Calculus of Variations and denoted by $\mathrm{BC} \int_{A} F(p, \phi \cdot q)$.

The following result shows that this new integral can be handled as a usual W-integral (see [9]). In order to see this, we consider the function $H:\left(K \times \mathbb{R}^{n}\right) \times \mathbb{R}^{m} \rightarrow B$ defined by

$$
H\left(k, e_{2} ; e_{1}\right)=F\left(k, e_{1} \cdot e_{2}\right) .
$$

$\mathrm{P}_{6}$. If $K$ is compact and the function $F$ satisfies conditions $\left(\mathrm{F}_{1}\right)$, $\left(\mathrm{F}_{2}\right)$ and $\left(\mathrm{F}_{3}\right)$, then also $H$ satisfies conditions $\left(\mathrm{F}_{1}\right),\left(\mathrm{F}_{2}\right)$ and $\left(\mathrm{F}_{3}\right)$ on any subset of the form $K \times B_{r}$, where

$$
B_{r}=\left\{e_{2} \in \mathbb{R}^{n}:\left\|e_{2}\right\| \leq r\right\}, \quad r>0 .
$$

Thus, as an immediate consequence of $\mathrm{P}_{6}$ and Theorem 3.4, we have the following existence result.

THeOREM 3.7. Suppose that $F$ satisfies conditions $\left(\mathrm{F}_{1}\right),\left(\mathrm{F}_{2}\right)$ and $\left(\mathrm{F}_{3}\right)$; the system $((p, q), \phi)$ is $\Gamma$-q.a., $\phi$ is $B V$ and $q$ is bounded; then $\Phi_{q}$ is q.a. and $B V$ on $A$.

REMARK 3.8. Note that if $q$ satisfies condition $(\gamma)$ and the couple $(p, \phi)$ is $\Gamma$-q.a., then the system $((p, q), \phi)$ is $\Gamma$-q.a.

4. Representation. In this section, for the sake of simplicity, we shall consider the following particular case of our setting. Note that this special case is quite natural since it always occurs in usual applications (see $\S 5$ ).

Note that we could obtain our representation theorem also in the general setting by strengthening the conditions on the function $\phi$. Instead, we prefer to consider the following restrictions:

- $A$ is a metric space

-there exists a subnet $\left(D_{t_{k}}\right)_{k \in \mathbb{N}}$ such that for every $k \in \mathbb{N}$

$$
I=\bigcup_{J \subset I} J, I \in D_{t_{k}}, J \in D_{t_{k+1}} \text { (i.e. a sequence of refinements); }
$$

-the intervals $\{I\}^{*}=\left\{I \in D_{t_{k}}, k \in \mathbb{N}\right\}$ are compact and connected; $-\lim _{k \rightarrow \infty} \max \left\{\operatorname{diam}(I), I \in D_{t_{k}}\right\}=0$.

Moreover we shall suppose that $E$ is a reflexive Banach space. 
We recall that an interval function $\phi:\{I\} \rightarrow E$ is said to be (0)quasi-additive ((0)-q.a.) ([17]) on $M \in \mathscr{M}$, if it satisfies condition (q.a.) where $s(J, I)$ is substituted by $s\left(J, I^{0}\right)$. Obviously (0)-q.a. is stronger than q.a.

In the present context the BC-integrals of the interval functions $\phi$ and $\|\phi\|$ can be extended to regular measures (see $[17,15,5])$. More precisely, if $\phi$ is (0)-q.a. and BV on $A$, then there exists a regular measure of bounded variation $\mu: \mathscr{B} \rightarrow E$ such that

$$
\mu(G)=\mathrm{BC} \int_{G} \phi \text { and }\|\mu\|(G)=\mathrm{BC} \int_{G}\|\phi\|, \quad G \in \mathscr{G},
$$

where $\|\mu\|$ denotes the variation of $\mu$. For further properties of $\mu$ and $\|\mu\|$ we refer to $[17,5,6,9]$.

Let $\phi, p$ and $q$ be the functions considered in $\S 3$. We define now the following sequences of step functions, $\eta_{k}: A \rightarrow \mathbb{R}^{n}, p_{k}: A \rightarrow K$, $q_{k}: A \rightarrow \mathbb{R}^{m}, k \in \mathbb{N}$

$$
\begin{aligned}
& \eta_{k}(a)= \begin{cases}\frac{\mu(I)}{\|\mu\|(I)}, & a \in I^{0}, I \in D_{t_{k}}, \quad\|\mu\|(I) \neq 0, \\
0, & \text { otherwise }\end{cases} \\
& p_{k}(a)= \begin{cases}p(I), & a \in I^{0}, I \in D_{t_{k}}, \\
p_{0}, & \text { otherwise }\end{cases} \\
& q_{k}(a)= \begin{cases}q(I), & a \in I^{0}, I \in D_{t_{k}} \\
q_{0}, & \text { otherwise }\end{cases}
\end{aligned}
$$

where $p_{0} \in K$ and $q_{0} \in \mathbb{R}^{m}$ are fixed.

As a consequence of a well-known convergence theorem for martingales, in $[6,7]$ we have proved the following result.

$\mathrm{P}_{7}$. If $\phi$ is (0)-q.a. and $B V$ on $A$, then $\eta_{k} \rightarrow d \mu / d\|\mu\| \mu$-almost everywhere, where $d \mu / d\|\mu\|$ denotes the Radon-Nikodym derivative.

In the following we will make use of the condition

(c) there exists a $\mu$-measurable function $\pi: A \rightarrow K$ such that $p_{k} \rightarrow \pi$ $\mu$-almost everywhere.

Note that if $p$ satisfies condition $(\gamma)$ then (c) holds; but this result is still valid under weaker assumptions on $p$, as $\S 5$ shows.

Finally the following representation result can be proved in an analogous way to Propositions 2, 3 and Theorem 1 of [6], taking into account $\mathrm{P}_{6}$.

Theorem 4.1. Suppose that $K$ is compact and $F$ satisfies conditions $\left(\mathrm{F}_{1}\right),\left(\mathrm{F}_{2}\right)$ and $\left(\mathrm{F}_{3}\right)$; the functions $((p, q), \phi)$ are $\Gamma$-q.a., $\phi$ is 
(0)-q.a. and BV on A, p, q satisfy condition ${ }^{3}$ (c) and $q$ is bounded, then

$$
\mathrm{BC} \int_{G} F(p, \phi \cdot q) \equiv \int_{G} F\left(\pi(a), \frac{d \mu}{d\|\mu\|}(a) \cdot q(a)\right) d\|\mu\|, \quad G \in \mathscr{G} .
$$

Note that in the absence of the weight $q$ the compactness of $K$ can be omitted and the function $\phi$ can be taken with values in $E$.

REMARK 4.2. In the previous theorem the function $\phi$ is (0)-q.a. and satisfies, together with $p$, condition ( $\Gamma$-q.a.). Thus it could be natural to introduce a new condition of $\Gamma$-(0)-quasi additivity ( $\Gamma$-(0)-q.a.); i.e. we say that the couple is $\Gamma$-(0)-q.a. if condition ( $\Gamma$-q.a.) holds, where $\Gamma_{I}$ is substituted by $\Gamma_{I^{0}}$ and $s(J, I)$ by $s\left(J, I^{0}\right)$. However note that these two conditions are equivalent:

(1) $(p, \phi)$ is $\Gamma$-q.a. and $\phi$ is (0)-q.a. on $A$,

(2) $(p, \phi)$ is $\Gamma$ - $(0)$-q.a.

In fact it is easy to see that (1) implies (2) with $\Gamma_{I^{0}}=\left\{J \in \Gamma_{I}: J \subset I^{0}\right\}$ and (2) implies (1) with $\Gamma_{I} \equiv \Gamma_{I^{0}}$.

\section{Applications.}

5.1. The parametric W-integral over a $B V$ curve. Let $x:[a, b] \rightarrow \mathbb{R}^{n}$ be a BV curve. As is well known $([\mathbf{1}, \mathbf{2 0}]), \lim _{\operatorname{ess}_{t \rightarrow c \pm}} x(t)=x(c \pm 0)$ exists for every $c \in[a, b]$ and if we take $E_{x}=\{c \in] a, b[: x(c)=$ $x(c+0)=x(c-0)\}$ and $S_{x}=\{c \in] a, b[: x(c+0) \neq x(c-0)\}$, then $[a, b]-E_{x}$ is a null set and $S_{x}$ is at most denumerable.

Let $\{I\}$ be the family of all closed sub-intervals of $[a, b]$ whose end-points belong to $E_{x}$ and let $\mathscr{D}_{x}$ be the collection of all finite subdivisions of the type $D=\left[I_{1}, \ldots, I_{N}\right]$ with $I_{i} \in\{I\}$ and $\bigcup_{1}^{N} i=$ $\left[\alpha_{1}, \alpha_{N+1}\right]$.

We consider the mesh function $\delta: \mathscr{D}_{x} \rightarrow \mathbb{R}$ defined by $\delta(D)=$ $\max \left\{\left(\alpha_{1}-a\right),\left(b-\alpha_{N+1}\right),|I|, I \in D\right\}$ which makes $\mathscr{D}_{x}$ a directed set.

Let $\Delta x(t)=x(t+0)-x(t-0)$. Observe now that for every $I \in\{I\}$, $\max _{t \in I}\|\Delta x(t)\|=m_{I}$ exists and we denote by $t_{I} \in I$ a point such that $\left\|\Delta x\left(t_{I}\right)\right\|=m_{I}$. If $m_{I}=0$ then $t_{I} \in I$ is arbitrary, otherwise $t_{I} \in I^{0}$.

Let $p_{x}:\{I\} \rightarrow \mathbb{R}^{n}$ be an interval function such that $p_{x}(I) \in \overline{\mathrm{co}} x_{\mathrm{ess}}(I)$ and consider the function $\Delta x:\{I\} \rightarrow \mathbb{R}^{\nu}$ with $1 \leq \nu \leq n$, defined by $\Delta x(I)=\Delta x([\alpha, \beta])=x(\beta-0)-x(\alpha+0)$.

\footnotetext{
${ }^{3}$ I.e. $p_{k} \rightarrow \pi$ and $q_{k} \rightarrow \xi \quad \mu$-almost everywhere.
} 
Note that $\Delta x$ may involve only $\nu$-components of the $n$-vector $x$. The following condition on the function $p_{x}$ will play a fundamental role in the existence result.

Definition 5.1. We say that $p_{x}$ satisfies condition $(\gamma)^{*}$ if

$(\gamma)^{*}$ for every $\varepsilon>0$ there exists $\eta=\eta(\varepsilon)>0$ such that for every $D_{0}=[I] \in \mathscr{D}_{x}$ with $\delta\left(D_{0}\right)<\eta$ there exists $\lambda=\lambda\left(\varepsilon, D_{0}\right)>0$ in such a way that, if $D=[J] \in \mathscr{D}_{x}$ with $\delta(D)<\lambda$, then for every $I \in D_{0}$, there exists $J_{I} \in D$ with $J_{I} \subset I, t_{I} \in J_{I}$ and $\left\|p_{x}(I)-p_{x}\left(J_{I}\right)\right\|<\varepsilon$.

LEMMA 5.2. If $p_{x}$ satisfies condition $(\gamma)^{*}$ then the couple $\left(p_{x}, \Delta x\right)$ is $\Gamma$-q.a. with respect to $\mathscr{D}_{x}$ and $\delta$.

Proof. Observe that if $S_{x}=\left\{t_{l}, l \in \mathbb{N}\right\}$ then $\sum_{l=1}^{\infty}\left\|\Delta x\left(t_{l}\right)\right\|$ $\leq V(x)<+\infty$, where $V$ denotes the generalized variation of $x$. Thus, given $\varepsilon>0$, let $k=k(\varepsilon) \in \mathbb{N}$ be such that

(1) $\sum_{l=k+1}^{\infty}\left\|\Delta x\left(t_{l}\right)\right\|<\varepsilon / 2$.

Then let $\delta_{1}=\delta_{1}(\varepsilon)>0$ be such that ${ }^{4}$

(2) if $|I|<\delta_{1}$ and $t_{l} \notin I, l=1, \ldots, k$, then $\omega_{\text {ess }}(x, I)<\varepsilon$; and moreover in such a way that

(3) $\left\|x\left(t_{l}+0\right)-x(t)\right\|<\varepsilon / 8 k$, for almost every $t \in\left[t_{l}, t_{l}+\delta_{1}\right]$, $\left\|x\left(t_{l}-0\right)-x(t)\right\|<\varepsilon / 8 k$, for almost every $t \in\left[t_{l}-\delta_{1}, t_{l}\right]$, $l=1, \ldots, k$.

Observe now that, without loss of generality, we can also suppose that $($ see $[1,20)]$

(4) $V(x,[a, \alpha])<\varepsilon / 4, V(x,[\beta, b])<\varepsilon / 4$ if $\alpha-a<\delta_{1}$ and $b-\beta<\delta_{1}$.

Now let $D_{0} \in \mathscr{D}_{x}$ be fixed with $\delta\left(D_{0}\right)<\min \left\{\delta_{1}, \eta,\left|t_{l}-t_{l^{\prime}}\right|, l \neq l^{\prime}\right.$, $\left.l, l^{\prime}=1, \ldots, k\right\}$ where $\eta=\eta(\varepsilon)>0$ is given by $(\gamma)^{*}$; and denote by $D_{0}=\left[I_{1}, \ldots, I_{N}\right], I_{i}=\left[\alpha_{i}, \alpha_{i+1}\right], i=1, \ldots, N$ its elements. Let $\delta_{2}=\delta_{2}\left(\varepsilon, D_{0}\right)>0$ be such that

(5) $\left\|x\left(\alpha_{i}\right)-x(t)\right\|<\varepsilon / 4 N$ for almost every $t \in\left[\alpha_{i}-\delta_{2}, \alpha_{i}+\delta_{2}\right]$, $i=1, \ldots, N+1$.

Let $\lambda=\lambda\left(\varepsilon, D_{0}\right)>0$ be given in $(\gamma)^{*}$ and denote by $D=[J] \in \mathscr{D}_{x}$ any finite division with $\delta(D)<\min \left\{\delta_{2}, \lambda, \alpha_{1}-a, b-\alpha_{N+1},|I|, I \in\right.$ $\left.D_{0}\right\}$. Now let $I=\left[\alpha_{i}, \alpha_{i+1}\right] \in D_{0}$ be fixed.

\footnotetext{
${ }^{4}$ Where $\omega_{\text {ess }}(x, I)$ denotes the essential oscillation of $x$ over $I$.
} 
Suppose first that $t_{l} \notin I, l=1, \ldots, k$. In this case we can take $\Gamma_{I}=\{J: J \subset I\}$, in fact by (2) we have that $\left\|p_{x}(I)-p_{x}(J)\right\|<$ $\omega_{\text {ess }}(x, I)<\varepsilon$, for every $J \in \Gamma_{I}$ and by (5)

(6)

$$
\begin{aligned}
\left\|\Delta x(I)-\sum_{J \subset I} \Delta x(J)\right\| & \leq\left\|x\left(\alpha_{i}\right)-x\left(\beta_{j}\right)\right\|+\left\|x\left(\gamma_{j}\right)-x\left(\alpha_{i+1}\right)\right\| \\
& \leq 2 \frac{\varepsilon}{4 N}=\frac{\varepsilon}{2 N}
\end{aligned}
$$

where $\bigcup_{J \subset I} J=\left[\beta_{j}, \gamma_{j}\right]$.

Otherwise $I$ contains only one point of the set $\left\{t_{l}, l=1, \ldots, k\right\}$ which coincides with $t_{I}$. Thus if we take $\Gamma_{I}=\left\{J_{I}\right\}$, where $J_{I}=$ $\left[\delta_{j}, \delta_{j+1}\right]$ is such that $t_{I} \in J_{I}$ and $\left\|p_{x}(I)-p_{x}\left(J_{I}\right)\right\|<\varepsilon$, then by (3) we have

$$
\begin{aligned}
\| \Delta x(I) & -\Delta x\left(J_{I}\right)\|\leq\| x\left(\alpha_{i}\right)-x\left(\delta_{j}\right)\|+\| x\left(\delta_{j+1}\right)-x\left(\alpha_{i+1}\right) \| \\
\leq & \left\|x\left(\alpha_{i}\right)-x\left(t_{I}-0\right)\right\|+\left\|x\left(t_{I}-0\right)-x\left(\delta_{j}\right)\right\| \\
& +\left\|x\left(\delta_{j+1}\right)-x\left(t_{I}+0\right)\right\|+\left\|x\left(t_{I}+0\right)-x\left(\alpha_{i+1}\right)\right\| \\
& \leq 4 \frac{\varepsilon}{8 k}=\frac{\varepsilon}{2 k} .
\end{aligned}
$$

Moreover, in this second case, again by force of (3) we also have

$$
\left\|\sum_{J \neq J_{I}} s(J, I) \Delta x(J)\right\| \leq\left\|x\left(\beta_{j}\right)-x\left(\delta_{j}\right)\right\|+\left\|x\left(\delta_{j+1}\right)-x\left(\gamma_{j}\right)\right\| \leq \frac{\varepsilon}{2 k} .
$$

Thus from (6), (7) and (8) we deduce that

$$
\sum_{I}\left\|\Delta x(I)-\sum_{J \in \Gamma_{I}} \Delta x(J)\right\|<\varepsilon
$$

and

$$
\sum_{I}\left\|\sum_{J \notin \Gamma_{I}} s(J, I) \Delta x(J)\right\|<\varepsilon .
$$

Finally, from (5) and (4) we have

$$
\begin{aligned}
\sum_{J} & {\left[1-\sum_{I} s(J, I)\right]\|\Delta x(J)\| } \\
& \leq V\left(x,\left[a, \alpha_{1}\right]\right)+N 2 \frac{\varepsilon}{4 N}+V\left(x,\left[\alpha_{N+1}, b\right]\right)<\varepsilon,
\end{aligned}
$$

which completes the proof. 
REMARK 5.3. Let us consider now the function $p_{x}=\left(p_{x}^{1}, \ldots, p_{x}^{n}\right)$ defined by

$$
p_{x}^{i}(I)=\lambda^{i} \inf \operatorname{ess}\left(x^{i}, I\right)+\left(1-\lambda^{i}\right) \sup \operatorname{ess}\left(x^{i}, I\right), \quad I \in\{I\},
$$

where $0 \leq \lambda^{i} \leq 1$ is fixed, $i=1, \ldots, n$.

Observe that the function $p_{x}$ satisfies condition $(\gamma)^{*}$. In order to see this, for sake of simplicity we take $n=1$. Given $\varepsilon>0$, let $k=$ $k(\varepsilon) \in \mathbb{N}$ be such that $\sum_{l=k+1}^{\infty}\left|\Delta x\left(t_{l}\right)\right|<\varepsilon / 2$, where $S_{x}=\left\{t_{l}, l \in \mathbb{N}\right\}$.

We denote by $m=\min \left\{\left|\Delta x\left(t_{l}\right)\right|, l=1, \ldots, k\right\}$ and by $\bar{\varepsilon}=$ $\min \{m / 4, \varepsilon / 4\}$; moreover let $\delta_{1}=\delta_{1}(\varepsilon)>0$ be such that

(2) if $|I|<\delta_{1}$ and $t_{l} \notin I, l=1, \ldots, k$, then $\omega_{\text {ess }}(x, I)<\varepsilon$ and

$$
\begin{array}{r}
\left|x\left(t_{l}+0\right)-x(t)\right|<\bar{\varepsilon}, \text { for almost all } t \in\left[t_{l}, t_{l}+\delta_{1}\right] \\
\left|x\left(t_{l}-0\right)-x(t)\right|<\bar{\varepsilon}, \text { for almost all } t \in\left[t_{l}-\delta_{1}, t_{l}\right], \\
l=1, \ldots, k
\end{array}
$$

We fix $D_{0}=\left[I_{1}, \ldots, I_{N}\right]$ and with $I_{i}=\left[\alpha_{i}, \alpha_{i+1}\right], i=1, \ldots, N$ and with $\delta\left(D_{0}\right)<\eta=\eta(\varepsilon)=\min \left\{\delta_{1},\left|t_{l}-t_{l^{\prime}}\right|, l \neq l^{\prime}, l, l^{\prime}=1, \ldots, k\right\}$ and let $D=[J] \in \mathscr{D}_{x}$ be given with $\delta(D)<\lambda=\lambda\left(\varepsilon, D_{0}\right)=$ $\min \left\{\left|\alpha_{i}-t_{l}\right|, i=1, \ldots, N+1, l=1, \ldots, k\right\}$. Now let $I=\left[\alpha_{i}, \alpha_{i+1}\right]$ be fixed.

In the case $t_{l} \notin I, l=1, \ldots, k$, by (2) we have for every $J \subset I$

(4) $\left|p_{x}(I)-p_{x}(J)\right|<\omega_{\text {ess }}(x, I)<\varepsilon$.

Conversely, suppose that $t_{l}=t_{I} \in I^{0}$, with $l \in\{1, \ldots, k\}$, and let $J_{I}=\left[\beta_{j}, \beta_{j+1}\right]$ be the interval of $D$ such that $t_{I} \in J^{0}$. By the choice of $\delta(D)$ we have $J \subset I$. Suppose now, without loss of generality, that $\inf \operatorname{ess}(x, I)=\inf \operatorname{ess}\left(x,\left[\alpha_{i}, t_{I}\right]\right)$; then by virtue of $(3)$ it is easy to see that also inf ess $\left(x, J_{I}\right)=\inf \operatorname{ess}\left(x,\left[\beta_{j}, t_{I}\right]\right)$. Thus again from (3) we have that

(5) $\left|\inf \operatorname{ess}(x, I)-\inf \operatorname{ess}\left(x, J_{I}\right)\right|<\varepsilon$. Analogously we can prove that

(6) $\left|\sup \operatorname{ess}(x, I)-\sup \operatorname{ess}\left(x, J_{I}\right)\right|<\varepsilon$ and from (5) and (6) it follows that

(7) $\left|p_{x}(I)-p_{x}\left(J_{I}\right)\right|<\varepsilon$.

REMARK 5.4. As a consequence of $P_{5}$ and Lemma 5.2, the function $\Delta x$ is q.a., but it is easy to prove that $\Delta x$ is also (0)-q.a. with respect to $\mathscr{D}_{x}$ and $\delta$ (see (5) of the proof of Lemma 5.2).

Let $\mu: \mathscr{B} \rightarrow \mathbb{R}^{n}$ be the measure which extends the BC-integral of $\Delta x$ (see $\S 4)$; then $\|\mu\|$ is exactly the variation-measure associated to $x$ (see [8]). Moreover, if $\left(p_{n}\right)_{n \in \mathbb{N}}$ denotes the sequence of step functions 
defined in $\S 4$, with respect to $p_{x}$, it can be proved that

(c) $p_{n} \rightarrow \pi \mu$-almost everywhere, with

$$
\begin{aligned}
\pi^{i}(t)= & \lambda^{i} \min \left(x^{i}(t+0), x^{i}(t-0)\right) \\
& +\left(1-\lambda^{i}\right) \max \left(x^{i}(t+0), x^{i}(t-0)\right), \quad i=1, \ldots, n .
\end{aligned}
$$

As a consequence of Theorems 3.4 and 4.1, by force of Lemma 5.1 and Remarks 5.3, 5.4 we have the following result.

THEOREM 5.5. Let $F: K \times \mathbb{R}^{n} \rightarrow \mathbb{R}, K \subset \mathbb{R}^{n}$ be a function satisfying $\left(\mathrm{F}_{1}\right),\left(\mathrm{F}_{2}\right)$ and $\left(\mathrm{F}_{3}\right)$ and let $x:[a, b] \rightarrow K$ be a $B V$ function. Then the interval function $\Phi:\{I\} \rightarrow \mathbb{R}$ defined by

$$
\Phi(I)=F\left(p_{x}(I), \Delta x(I)\right)
$$

where $p_{x}^{i}(I)=\lambda^{i} \inf \operatorname{ess}\left(x^{i}, I\right)+\left(1-\lambda^{i}\right) \sup \operatorname{ess}\left(x^{i}, I\right), i=1, \ldots, n$, is q.a. and $B V$ with respect to $\mathscr{D}_{x}$ and $\delta$; moreover

$$
\mathrm{BC} \int_{[a, b]} \Phi=\int_{a}^{b} F\left(\pi(t), \frac{d \mu}{d\|\mu\|}(t)\right) d\|\mu\| .
$$

In particular, if $x$ is absolutely continuous in the generalized sense, then

$$
\mathrm{BC} \int_{[a, b]} \Phi=\int_{a}^{b} F\left(x(t), x^{\prime}(t)\right) d t .
$$

Theorem 5.5 allows to define the W-integral over a BV curve obtaining an extension of the well-known results given for continuous BV-curves (see [20] for a survey). Moreover note that, since the function $p_{x}$ does not satisfy condition $(\gamma)$, Theorem 5.5 could not be proved as a consequence of the already known existence results for the W-integral.

Note that the results presented here improve the analogous ones given in [11]. For a different approach to a Weierstrass-type integral over a BV curve, see [19].

REMARK 5.6. The function $p_{x}$ of Remark 5.3 provides a choice in $\overline{c o} x(I)$ which works well. On the contrary the interval function $p_{x}:\{I\} \rightarrow \mathbb{R}$ defined by $p_{x}(I)=|I|^{-1} \int_{I} x(t) d t$ is not a good choice as the following example shows.

EXAMPLE 5.7. Let $x:[-1,1] \rightarrow[0,1]$ be the curve defined by $x(t)=0$ for $t \in[-1,0], x(t)=1$ for $t \in] 0,1]$ and consider the integrand $F:[0,1] \times \mathbb{R} \rightarrow \mathbb{R}$ defined by $F(k, x)=k|x|$.

Then the interval function $\Phi(I)=F\left(p_{x}(I), \Delta x(I)\right)$ is not BCintegrable. In fact note that $\Phi(I) \neq 0$ if and only if $0 \in I^{0}$, but in this 
case $\Phi(I)=|I \cap[0,1]| /|I|$ and this shows easily that $\lim _{|I| \rightarrow 0} \Phi(I)$ does not exist.

5.2. The weighted parametric $\mathrm{W}$-integral over a $B V$ curve. According to Remark 3.6, we shall introduce a weight in the $\mathrm{W}$-integral of $\S 5.1$.

In order to do this, besides the BV curve $x$, we consider a BV function $y:[a, b] \rightarrow \mathbb{R}^{n}$. We denote by $\mathscr{D}_{x, y}$ and $\delta$ the family of finite systems and the mesh introduced in $\S 5.1$ with regard to the curve $(x, y)$. Moreover, let $q_{y}:\{I\} \rightarrow \mathbb{R}^{n}$ be an interval function such that $q_{y}(I) \in \overline{\mathrm{co}} y_{\mathrm{ess}}(I)$.

As an application of Lemma 5.2 we have the following result.

LEMMA 5.7. If the function $\left(p_{x}, q_{y}\right)$ satisfies condition $(\gamma)^{*}$, then the system $\left(p_{x}, q_{y} ; \Delta x\right)$ is $\Gamma$-q.a. with respect to $\mathscr{D}_{x, y}$ and $\delta$.

By virtue of Lemma 5.7 and Remarks 5.3 and 5.4, as an application of Theorem 3.7, we have the following existence result.

THEOREM 5.8. Suppose that the function $F: K \times \mathbb{R}^{n} \rightarrow \mathbb{R}$, with $K \subset$ $\mathbb{R}^{n}$, satisfies conditions $\left(\mathrm{F}_{1}\right),\left(\mathrm{F}_{2}\right)$ and $\left(\mathrm{F}_{3}\right) ;$ moreover let $x:[a, b] \rightarrow$ $K$ and $y:[a, b] \rightarrow \mathbb{R}$ be two $B V$ functions. Then the function $\Phi_{q}:\{I\}$ $\rightarrow \mathbb{R}$ defined by

$$
\Phi_{q}(I)=F\left(p_{x}(I), \Delta x(I) \cdot q_{x}(I)\right)
$$

where

$$
\begin{aligned}
& p_{x}^{i}(I)=\alpha^{i} \inf \operatorname{ess}\left(x^{i}, I\right)+\left(1-\alpha^{i}\right) \sup \operatorname{ess}\left(x^{i}, I\right), \\
& q_{y}^{i}(I)=\beta^{i} \inf \operatorname{ess}\left(y^{i}, I\right)+\left(1-\beta^{i}\right) \sup \operatorname{ess}\left(y^{i}, I\right), \alpha^{i}, \beta^{i} \in[0,1], \\
& \quad i=1, \ldots, n
\end{aligned}
$$

is q.a. and $B V$ with respect to $\mathscr{D}_{x, y}$ and $\delta$. Moreover we have

$$
\mathrm{BC} \int_{[a, b]} \Phi_{q}=\int_{a}^{b} F\left(\pi(t), \frac{d \mu}{d\|\mu\|}(t) \cdot \xi(t)\right) d\|\mu\|
$$

where

$$
\begin{aligned}
& \pi^{i}(t)=\alpha^{i} \min \left(x^{i}(t+0), x^{i}(t-0)\right) \\
& +\left(1-\alpha^{i}\right) \max \left(x^{i}(t+0), x^{i}(t-0)\right) \quad \text { and } \\
& \xi^{i}(t)=\beta^{i} \min \left(y^{i}(t+0), y^{i}(t-0)\right) \\
& +\left(1-\beta^{i}\right) \max \left(y^{i}(t+0), y^{i}(t-0)\right), \quad i=1, \ldots, n .
\end{aligned}
$$


In the particular case that $x$ is absolutely continuous in the generalized sense, then we have

$$
\mathrm{BC} \int_{[a, b]} \Phi_{q}=\int_{a}^{b} F\left(x(t), x^{\prime}(t) \cdot \xi(t)\right) d t .
$$

REMARK 5.9. If $y:[a, b] \rightarrow \mathbb{R}^{n}$ is continuous then any function $q_{y}:\{I\} \rightarrow \mathbb{R}^{n}$ such that $q_{y}(I) \in \overline{\operatorname{co}} y(I)=y(I)$ satisfies condition $(\gamma)$. Therefore also in this case a result analogous to Theorem 5.7 holds. Moreover note that now we have $\xi(t)=y(t)$.

The weighted W-integral presented in this section extends the weighted generalized length of a parametric BV curve (see $[1,20,3])$. Note that here the weight is not necessarily continuous and positive.

\section{REFERENCES}

[1] M. Boni, Variazione generalizzata con peso e quasi additività, Atti Sem. Mat. Fis. Univ. Modena, 25 (1976), 195-210.

[2] M. Boni and P. Brandi, Teoremi di esistenza per l'integrale del Calcolo delle Variazioni nel caso ordinario, Atti Sem. Mat. Fis. Univ. Modena, 23 (1974), 308-327.

[3] P. Brandi, Variazione, classica e generalizzata, rispetto ad una misura con derivata totalmente misurabile, Atti Sem. Mat. Fis. Univ. Modena, 28 (1979), 63-85.

[4] P. Brandi and A. Salvadori, Sull'integrale debole alla Burkill-Cesari, Atti Sem. Mat. Fis. Univ. Modena, 27 (1978), 14-38.

[5] _ Sull'estensione dell'integrale debole alla Burkill-Cesari ad una misura, Rend. Circ. Mat. Palermo, 30 (1981), 207-234.

[6] __ Un teorema di rappresentazione per l'integrale parametrico del Calcolo delle Variazioni alla Weierstrass, Ann. Mat. Pura Appl., 124 (1980), 39-58.

[7] _ Martingale ed integrale alla Burkill-Cesari, Rend. Accad. Naz. Lincei, 67 (1979), 197-203.

[8] _ Sull'area generalizzata, Atti Sem. Mat. Fis. Univ. Modena, 24 (1979), 33-62.

[9] _ The non parametric integral of the Calculus of Variations as a Weierstrass integral. I-Existence and representation, J. Math. Anal. Appl., 107 (1985), 6795.

[10] _ The non parametric integral of the Calculus of Variations as a Weierstrass integral. II-Some applications, J. Math. Anal. Appl., 112 (1985), 290-313.

[11] __ Existence, semicontinuity and representation for the integrals of the Calculus of Variations. The BV case, Atti Convegno celebrativo I centenario Circolo Matematico di Palermo, (1984), 447-462.

[12] __ L'integrale del Calcolo delle Variazioni alla Weierstrass lungo curve BV e confronto con i funzionali integrali di Lebesgue e Serrin, Atti Sem. Mat. Fis. Univ. Modena, 35 (1987), 319-325.

[13] __ On the non-parametric integral over a BV surface, J. Nonlinear Anal., 13 (1989), 1127-1137. 
[14] P. Brandi and A. Salvadori, On the lower semicontinuity of certain integrals of the calculus of variations, J. Math. Anal. Appl., 144 (1989), 183-205.

[15] J. C. Breckenridge, Burkill-Cesari integrals of quasi additive interval functions, Pacific. J. Math., 37 (1971), 635-654.

[16] L. Cesari, Quasi additive set functions and the concept of integral over a variety, Trans. Amer. Math. Soc., 102 (1962), 94-113.

[17] __ Extension problem for quasi additive set functions and Radon-Nikodym derivatives, Trans. Amer. Math. Soc., 102 (1962), 114-146.

[18] P. J. Kaiser, Length and variation with respect to a measure, Atti Sem. Mat. Fis. Univ. Modena, 24 (1976), 221-235.

[19] M. Ragni, L'integrale parametrico del calcolo delle variazioni alla Weierstrass in seuso generalizzato, Le Matematiche, 34 (1979), 21-38.

[20] A. Salvadori, Sulla convergenza in lunghezza in senso generalizzato con peso per una successione di curve parametriche, Rend. Circ. Mat. Palermo, 26 (1977), 195-228.

[21] C. Vinti, Nonlinear integration and Weierstrass integral over a manifold, connection with theorems on martingales, J. Optim. Theory Appl., 41 (1983), 213-237.

[22] G. Warner, The Burkill-Cesari integral, Duke Math. J., 35 (1968), 61-78.

[23] _ The generalized Weierstrass-type integral $\int f(\xi, \phi)$, Ann. Scuola Norm. Sup. Pisa, 22 (1968), 163-192.

Received October 20, 1988. This research has been carried out within the GNAFACNR.

DEPARTMENT OF ENGINEERING

UNIVERSITY OF L'AQUILA

67100 L'AQUila, ITALY

AND

University of Perugia

06100 Perugia, ItAly 



\section{PACIFIC JOURNAL OF MATHEMATICS EDITORS}

\author{
V. S. VARADARAJAN \\ (Managing Editor) \\ University of California \\ Los Angeles, CA 90024-1555-05 \\ Herbert Clemens \\ University of Utah \\ Salt Lake City, UT 84112 \\ Thomas ENRIGHT \\ University of California, San Diego \\ La Jolla, CA 92093
}

\section{R. FINN}

Stanford University

Stanford, CA 94305

Hermann FlaschKa

University of Arizona

Tucson, AZ 85721

VAUGHAN F. R. Jones

University of California

Berkeley, CA 94720

STEVEN KeRCKHOFF

Stanford University

Stanford, CA 94305
C. C. MOORE

University of California

Berkeley, CA 94720

Martin ScharlemanN

University of California

Santa Barbara, CA 93106

HAROLd STARK

University of California, San Diego

La Jolla, CA 92093

\section{ASSOCIATE EDITORS}
R. ARENS
E. F. BECKENBACH
B. H. NeumanN
F. Wolf
K. YoshidA (1906-1982)
(1904-1989)

\section{SUPPORTING INSTITUTIONS}
UNIVERSITY OF ARIZONA
UNIVERSITY OF BRITISH COLUMBIA
CALIFORNIA INSTITUTE OF TECHNOLOGY
UNIVERSITY OF CALIFORNIA
MONTANA STATE UNIVERSITY
UNIVERSITY OF NEVADA, RENO
NEW MEXICO STATE UNIVERSITY
OREGON STATE UNIVERSITY
UNIVERSITY OF OREGON
UNIVERSITY OF SOUTHERN CALIFORNIA
STANFORD UNIVERSITY
UNIVERSITY OF HAWAII
UNIVERSITY OF TOKYO
UNIVERSITY OF UTAH
WASHINGTON STATE UNIVERSITY
UNIVERSITY OF WASHINGTON 


\section{Pacific Journal of Mathematics}

Vol. 146, No. $1 \quad$ November, 1990

Primo Brandi and Anna Salvadori, A quasi-additivity type condition and

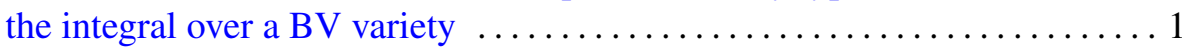

Dong M. Chung, Chull Park and David Lee Skoug, Operator-valued

Feynman integrals via conditional Feynman integrals ..............21

Paul Jolissaint, Index for pairs of finite von Neumann algebras . .........43

Miodrag Mateljević and Miroslav Pavlović, Multipliers of $H^{p}$ and

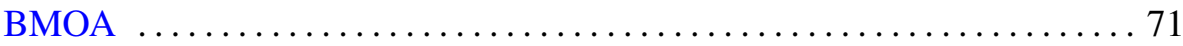

Himadri Kumar Mukerjee, Poincaré cobordism exact sequences and

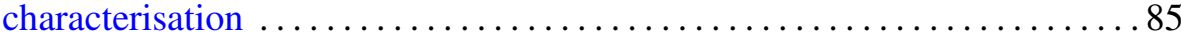

Thomas H. Otway, The coupled Yang-Mills-Dirac equations for differential

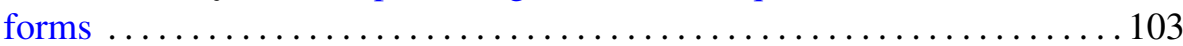

Sechiko Takahashi, Nevanlinna parametrizations for the extended

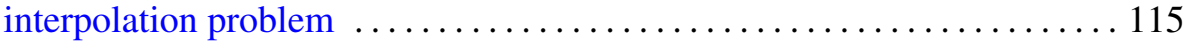

P. C. Trombi, Uniform asymptotics for real reductive Lie groups ........ 131 